\title{
Visual Simulation of Modern Supply Chain Based on MR Virtual Reality Technology
}

\author{
Jin Yang $(\mathbb{D}$ \\ Wuhan Technical College of Communicaitions, Wuhan 430065, China \\ Correspondence should be addressed to Jin Yang; tobbyyoung@whtcc.edu.cn
}

Received 15 December 2021; Revised 4 January 2022; Accepted 15 January 2022; Published 8 February 2022

Academic Editor: Qiangyi Li

Copyright ( $\odot 2022$ Jin Yang. This is an open access article distributed under the Creative Commons Attribution License, which permits unrestricted use, distribution, and reproduction in any medium, provided the original work is properly cited.

\begin{abstract}
In order to improve the management and operation efficiency of the modern supply chain, this paper combines the MR virtual reality technology to carry out the visual simulation of the modern supply chain. Moreover, this paper uses MR virtual simulation technology to perform intelligent perception of supply chain transportation equipment and transportation objects, recognize its shape and the force it receives, and ensure that there is no damage during the transportation process. The visual simulation of supply chain is realized by real-time dynamic display based on geometric model. Moreover, this paper combines the MR virtual reality technology to define the geometric and kinematic characteristics of the modeled object, then encapsulates these characteristics into the object class, and defines the behavior characteristics to establish an object class library. Finally, the experimental research verifies that the modern supply chain visual simulation technology based on MR virtual reality technology has a good simulation effect in the supply chain system.
\end{abstract}

\section{Introduction}

Supply chain information visualization is not a new concept, and it is a means for companies to manage their supply chain through informatization. After consulting some domestic and foreign related materials, it is not found that the existing related literature has a clear definition of supply chain information visualization. The current research on the visualization of supply chain information refers to a high degree of information sharing among enterprises at various nodes of the supply chain, and various types of information flow in a reasonable and rapid manner in accordance with the requirements of the enterprise in each link of the supply chain. Moreover, the information is transparent to the corresponding management personnel of the enterprise, thereby assisting the decision makers of the enterprise to make the right decision at the right time [1]. The visualized supply chain also refers to a supply chain with a high degree of information sharing. "Highly shared" and "visualization" do not refer to the unlimited sharing and opening of information, but the opening and sharing of information according to the actual business needs and personnel responsibilities of the enterprise [2]. For example, the company's decision-makers pay more attention to the realtime inventory level and supply and sales of the entire group. Obviously, this information is not suitable for opening to other noncorporate decision-makers. The specific business executives within the enterprise may pay more attention to the progress of specific services, such as the status of purchase orders and the expected arrival or time, and the inventory of a specific product in a certain area of the factory [3].

Supply chain members receive information from different sources and channels at all times in their daily production and operation activities. This information is an important resource necessary for enterprises to make various production and operation decisions. The quality of this information directly affects the level of corporate decisionmaking, which further affects corporate earnings. With the continuous development of IT technology, the role of information has become more and more obvious, and supply chain members are increasingly relying on various information to avoid risks and create value. To make good use of information, this precious resource, we must first clarify the 
characteristics and attributes of supply chain information. There are many types of information in the supply chain, and each supply chain node is faced with different information from different channels. For example, the information channel of a retailer involves customers, suppliers, the external environment of the enterprise, competitors, and partners. Information from different channels is diverse. For example, supplier information includes production information, order status information, inventory information, product information, supply capability information, and technological progress information. Information on the supply chain guides the movement of logistics. Effective use of the correct information to improve the competitive advantage of an enterprise is one of the strategies of an enterprise. In a sense, the supply chain is actually a piece of information used to meet customer needs.

This paper combines the MR virtual reality technology to carry out the visual simulation of the modern supply chain, improve the management and operation efficiency of the modern supply chain, and provide a theoretical reference for the subsequent research on the visual management of the supply chain.

\section{Related Work}

Literature [4] gives a quantitative model of the value of demand information in the 1-1 secondary supply chain under the condition of uncertain terminal demand information, and proves that sharing terminal market demand information in the supply chain can effectively suppress the bullwhip effect and reduce manufacturers' inventory levels and their costs, but retailers can hardly benefit directly from them. Literature [5] compares the impact of high-level and low-level demand information sharing on supply chain costs, and proves that sharing high-level demand information can greatly reduce supply chain costs. Literature [6] studied the impact of shared demand information accuracy on the improvement of manufacturers' inventory holdings, and found that if manufacturers choose an accurate demand information system, even if the required cost is low, they will not choose to share inventory information completely. Improving inventory holdings can effectively manage the competition among retailers. When retailers are fully subdivided, manufacturers can also obtain certain residual profits.

Literature [7] established a single-project multi-stage, shared demand information, and customer demand autoregressive joint moving average process (ARIMA) system, and found that the prediction model adopted by the system members is the main reason for the bullwhip effect. The availability of information does not have a beneficial effect on supply chain costs, but will increase the complexity and cost of the system. Literature [8] analyzed how to encourage competing retailers to share information vertically with suppliers in the 1-N secondary supply chain, and found that simple sharing of information does not always benefit participants, and terminal sales information is in the 1-1 vertical. In addition to the "direct effect" in the transmission, there may also be a "leakage effect," that is, competitors can infer the private information of the information sharer by observing the changes in the information sharer's behavior, thereby adjusting their decision-making behavior to benefit.

The value of upstream corporate information visualization has attracted people's attention, but upstream corporate information sharing also plays an indispensable role in supply chain management. Literature [9] compares whether retailers in the 1-1 secondary supply chain understand the impact of supplier inventory strategies and service levels on supply chain performance, and finds that supplier cooperation can improve the stability and service level of the supply chain, thereby improving its performance.

Literature [10] introduces two technologies for realizing in-transit logistics visualization-radio frequency identification technology and network GPS technology. On this basis, it analyzes and compares them, and puts forward corresponding suggestions. Thürer et al. [11] start with the analysis of the characteristics of GIS visualization technology, taking the structure of the radio and television GIS-MIS system and GeoWind0Ws system, spatial data processing, database construction, and the realization of functions in the collection and query of radio, film and television information, and statistics as examples. It introduced the key technology of GIS visualization technology in the management information system interface development, and gave the corresponding constructive results, and finally showed the application prospect of GIS visualization technology in the management information system. Sun et al. [12] believe that RF-based data collection methods can obtain information quickly and accurately, and can effectively eliminate the "bullwhip effect" in the logistics process. Davis et al. [13] introduced the application of radio frequency identification (RFID) technology to track the work flow of materials in transit, and pointed out its defects. The visibility system of in-transit materials composed of RFID, global positioning system (GPS), geographic information system (GIS), computer network, and satellite communication is analyzed. Yang et al. [14] found that inventory visualization can reduce inventory turnover time by more than $20 \%$, and inventory can also be reduced by more than 20\%. Coatney and Poliak [15] believe that companies that invest in supply chain visualization solutions can respond more quickly to demand and radically reduce costs. Hawkins [16] defines visualization as: by seeing the logistics process, resources, and customer requirements, so as to provide the necessary knowledge support for effective decision-making. Literature [17] described the trend of global supply chain visualization, and $91 \%$ of the respondents mentioned that they would use supply chain visualization tools to improve their global trading system. Companies that use a global supply chain visualization platform can reduce costs and lead time by 2 times.

Supply chain visualization is an emerging technology. It has attracted extensive attention from foreign scholars in optimizing the entire supply chain operation and improving supply chain performance. Currently, supply chain visualization solutions have been proposed from the aspects of inventory and logistics, but not very systematic and practical [18]; domestic scholars have done little research on visualization, mainly 
focusing on logistics and transportation. The application methods are mainly to use radio frequency technology RFID and GIS//GPS systems to track the physical location. In this way, the transportation link is visualized, and it is not extended to the entire supply chain environment, which is far behind foreign countries. However, articles on the application of supply chain visualization technology to supply chain performance evaluation have not been retrieved. Therefore, supply chain visualization technology is used in supply chain performance evaluation, and real-time dynamic display of supply chain performance is worthy of in-depth study.

\section{Supply Chain Transportation Object Structure Simulation Combined with MR Virtual Simulation}

In order to realize the visual simulation of modern supply chain, the visualization in this paper is different from other supply chain visualization systems. This paper uses MR virtual simulation technology to carry out the intelligent perception of supply chain transportation equipment and transportation objects, recognize its shape and the force it receives, and ensure that there is no damage during the transportation process.

In practice, the specific structure of the problem can be used to reduce the computational burden of the supply chain system.

$$
\in(u)=\left\{\begin{array}{c}
u_{1,1} \\
u_{2,2} \\
u_{3,3} \\
u_{2,3}+u_{3,2} \\
u_{3,1}+u_{1,3} \\
u_{1,2}+u_{2,1}
\end{array}\right\}
$$

According to the previous notation convention, $\epsilon=\left\{\epsilon_{i j}\right\}$ is a strain matrix. This matrix will no longer be explicitly needed, so we keep $\epsilon$ as the strain vector, as shown in (1). Now, we compare the fourth-order tensor of elastic coefficients into a matrix, and compare the fourth-order tensor of elastic coefficients into the matrix:

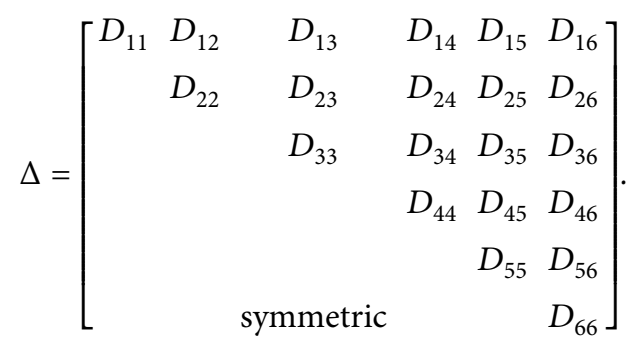

For elastic tensors, the algorithm compresses indexes $i$ and $j$ to I, and compresses $k$ and I to J. If there is a need for the value of I in the first column, the algorithm reads $i$ from the second column and $j$ from the third column. Correspondingly, if $J$ is obtained from the first column, then $k$ is read in the second column and $l$ is read in the third column. With $\in$ and $D$ (the same symbolic form as the strain vector), the virtual perceptual force vector is defined as

$$
\sigma=\left\{\begin{array}{l}
\sigma_{11} \\
\sigma_{22} \\
\sigma_{33} \\
\sigma_{23} \\
\sigma_{31} \\
\sigma_{12}
\end{array}\right\}=D \in(u)
$$

This is commonly referred to as Voight notation. We noticed from (1) that half of the factors are deleted from the shear component (term containing the cross derivative). This has been explained in (3), because it is now that each cut term does not contribute to any half, which is the opposite of the two identical contributions of the term containing the factor. Moreover, all these new definitions make it possible to write

$$
a(w, u)=\int_{\Omega} \in(w)^{T} D \in(u) \mathrm{d} \Omega .
$$

In addition, it can be extended in the following ways:

$$
\in\left(N_{A} e_{i}\right)=B_{A} e_{i} .
$$

Here,

$$
B_{A}=\left[\begin{array}{ccc}
N_{A, 1} & 0 & 0 \\
0 & N_{A, 2} & 0 \\
0 & 0 & N_{A, 3} \\
0 & N_{A, 3} & N_{A, 2} \\
N_{A, 3} & 0 & N_{A, 1} \\
N_{A, 2} & N_{A, 1} & 0
\end{array}\right] .
$$

Therefore, the term of the overall stiffness matrix can be rewritten as

$$
K_{P Q}=e_{i}^{T} \int_{\Omega} B_{A}^{T} D B_{B} \mathrm{~d} \Omega e_{j} .
$$

The sparse global stiffness matrix and force vector are constructed by traversing the unit and constructing a dense local stiffness matrix and force vector, which are then assembled into the global system. It has $d$ spatial dimensions (that is, the degrees of freedom of each control variable) and $n_{\text {en }}$ local shape functions, and calculates the terms of the local stiffness matrix on unit $\Omega^{e}[19]$.

$$
K_{p q}=e_{i}^{T} \int_{\Omega^{e}} B_{a}^{T} D B_{b} \mathrm{~d} \Omega e_{j} .
$$

Here,

$$
\begin{aligned}
& p=d(a-1)+i, \\
& q=d(b-1)+j .
\end{aligned}
$$

Similarly, the local force vector of the element is given by

$$
f_{p}^{e}=\int_{\Omega^{e}} N_{a} f_{i} d \Omega+\int_{\Gamma_{h_{i}}^{e}} N_{a} h_{i} d \Omega-\sum_{q=1}^{d \cdot n_{\mathrm{en}}} k_{p q}^{e} g_{q}^{e} .
$$


Here, $\Gamma_{h_{i}}^{e}$ is the intersection of the cell boundaries in $\Gamma_{h_{i}}$, and $q=d(b-1)+j$ and $Q=L M(j, b, e)$. Of course, in fact, the Gauss quadrature rule is used to calculate the integral here.

The increase in the index may seem a bit confusing at first. The IEN has already been introduced, which associates the local shape function number of a given element number with its corresponding global shape function number, denoting the ID. It associates the global shape function number and degree of freedom number with the global equation number, denoted as LM. It combines the first two and associates the element number, degree of freedom number, and local shape function number with the corresponding global equation number. Without defining any new array, this paper uses (9) to connect the local shape function number with the local equation number. In practice, the LM array is usually overloaded so that it can hold two indexes, namely, the element number and the local equation number, or three indexes, the element number, the local shape function number, and the degree of freedom number. Then, the algorithm returns the local equation numbers in these two cases, so that

$$
\operatorname{LM}(p, e)=\operatorname{LM}(i, a, e)
$$

where $p$ is shown in (9).

The pseudo code of a basis function routine proposed in this paper, and it will input a unit number and an integration point in the unit, and return the basis function. Their derivatives with respect to physical coordinates and the Jacobian determinant of the mapping are calculated at the integration point in the physical domain. In the following, this function will be used to establish and combine the local stiffness matrix and load vector required for uniform linear elastic solid analysis.

In order to increase the calculation speed, it is necessary to control the decomposition level. In order to fine-tune the stiffness matrix of the decomposition calculation, a stiffness correction algorithm based on the cut surface of the supply chain transportation object is proposed.

In the CAD system, the intersection judgment is extremely time-consuming in the three-dimensional space, and the calculation of the system stiffness matrix is more time-consuming. In order to solve this problem, it is necessary to conduct in-depth research on the cutting unit of transported objects in the supply chain. From the perspective of stiffness matrix calculation, effective and complete parameter elements can be directly obtained by calculation. The remaining invalid parameter units have no effect on the stiffness of the system, while the supply chain transportation object cutting parameter unit has a greater impact on the value of the stiffness matrix and is directly used. This operation directly leads to the distortion of the calculation accuracy.

From a macro point of view, this approximate concept reflects the degree of influence of the effective space on the overall stiffness matrix on the cutting parameter unit of the supply chain transportation object. If it is small, it will be discarded, but the stiffness is softer than the real situation. If an appropriate coefficient is selected to adjust the effective and ineffective parts before the split ratio, and then the stiffness of the effective part is fed back to the system stiffness, the softening of the stiffness can be effectively improved. It is difficult to accurately define the effective area and invalid area of the supply chain transportation object cutting unit due to the complexity of the position of the supply chain transportation object cutting surface and the accuracy of the intersection. This paper considers the 8 vertices $P$ and the center of gravity $P$ of the bottom parameter unit of the supply chain transportation object cutting corresponding to the actual space coordinates and the orientation of the supply chain transportation object cutting surface, and judges the effective area weight based on the relative position. [20]:

The following restrictions are imposed on the nine points

$$
W_{P_{m}}+\sum_{i=1}^{8} W_{P_{v}^{j}}=1
$$

Because the position of the center of gravity is more important, the algorithm takes the weight 0.2 , and the weight of the remaining vertices is 0.1 , and the effective area of the element is (sub-element stiffness correction coefficient):

$$
\lambda_{r}=\alpha W_{P_{m}}+\sum_{i=1}^{8} W_{P_{v}^{j}} \alpha, \beta= \begin{cases}1, & P_{m} \text { or } P_{v} \text { are in effective area, } \\ 0, & \text { otherwise }\end{cases}
$$

Finally, the modified stiffness matrix expression of the bottom-layer unit of the objects in each supply chain is obtained as $\lambda_{r} K_{\text {local }}$. The sub-cell stiffness $K_{\text {local }}$ can be directly obtained from the complete element stiffness generating formula.

Compared with the complete model, in the isogeometric analysis of the supply chain transportation object cutting model, the application of the cutting boundary conditions of the supply chain transportation object has also caused many problems. The fundamental reason is that the parameter domain has changed. The problem has been briefly introduced before, and some solutions are discussed in detail below. For isogeometric analysis, the loading of boundary conditions can be divided into two aspects: the application of essential boundary conditions (Dirichlet boundary conditions) and the application of natural boundary conditions (Neumann boundary conditions). Due to the limitation of the length of the article, this paper only takes linear elastic statics as an example to illustrate the natural boundary loading method of the supply chain transportation object cutting model.

In the isogeometric analysis process of the cutting model of the supply chain transportation object, the boundary is loaded on the cutting surface of the nonsupply chain transportation object, and the natural boundary can still be directly introduced into the integral formula calculation. If it is loaded on the cutting surface of the transportation object in the supply chain, because the control point of the cutting 
model of the supply chain transportation object cannot fully express the cutting surface of the transportation object in the supply chain, the integral cannot be directly run.

Because the degree of freedom of the cutting model of the supply chain transportation object in IGA is determined by its control point, it is not located at the control point of the cutting surface of the supply chain transportation object. At this time, an equivalent loading method is proposed. The specific operation is to add an equivalent force distribution field to the original parameter domain of the supply chain transportation object cutting entity control point to replace the loading on the supply chain transportation object cutting surface.

As shown in Figure 1, an example of a trimmed surface of a two-dimensional supply chain transportation object is provided. The red dot in the figure represents the control point of the cutting surface of the object transported by the supply chain, and the black dot represents the control point of the cutting curve of the object transported by the supply chain. It can be observed that the two are obviously different, and there is no direct mathematical relationship between them. In this way, if the force boundary is applied in the traditional FEM loading method, the loading force will be equivalent to the red control point, but the degree of freedom of the model is determined by the original control point (black). On the whole, the effect of this method is not effective, in other words, the force boundary conditions are not accurately applied. In order to obtain accurate and effective simulation data, equal power must be applied to the original voxel control points.

The equal effect vector is obtained by integrating the force boundary on the trimmed surface of the transportation object in the supply chain. When physical strength is not considered, it is expressed as the following form:

$$
f_{e}=\iint_{\Gamma} N^{T} \bar{F} \mathrm{~d} \Gamma \text {. }
$$

The equivalent power vector on the control point can be expressed by the following formula:

$$
\left\{\begin{array}{l}
f_{x} \\
f_{y} \\
f_{z}
\end{array}\right\}=\sum_{k=1}^{n} N_{i}^{\mathrm{T}} \bar{F}|J| W_{k} .
$$

Here, $N$ is the basis function of the cutting curve/surface of the object transported in the supply chain, and $\bar{F}$ is the force distribution function of the cutting boundary of the object transported in the supply chain. $|J|$ is the modulus $\|(\partial S / \partial u) \times$ $(\partial S / \partial v) \|$ of the normal vector of the trimmed surface of the transported object in the supply chain at the numerical integration point, or the modulus $\|(\partial C / \partial u)\|$ of the trimming curve of the transported object in the supply chain, and $\mathrm{W}$ represents the weight of the integration point at the trimming boundary of the transported object in the supply chain.

Because $N$ is not the basis function of the basic voxel, the force vector directly calculated by this method cannot correspond to the system equation.

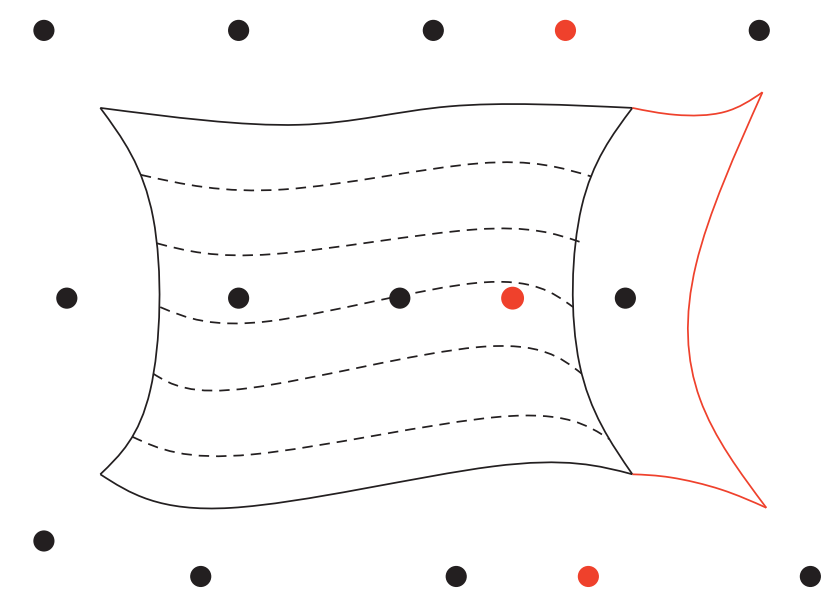

FIGURE 1: Distribution of control points on the trimmed surface of objects transported in the supply chain.

Although the clipping boundary I of supply chain transportation objects is represented separately, it is located in the space represented by the original basic voxel $\Omega$, that is, $\Gamma \in \Omega$. For the trimmed surface of the transportation object in the supply chain, a point $\left[x_{0}, y_{0}, z_{0}\right]^{T}$ in the physical space can obtain the corresponding parameter point $\left(u^{C}\right),\left(u^{S}, v^{S}\right)$ from the trimming boundary parameter space and the original parameter space of the transportation object in the supply chain. In view of this, the equivalent power vector in formula (15) can be rewritten as [21]

$$
\left\{\begin{array}{l}
f_{x} \\
f_{y} \\
f_{z}
\end{array}\right\}=\sum_{k=1}^{n} N_{i}^{S}\left(u^{S}, v^{S}\right) \bar{F}|J| W_{k} .
$$

Here, $\left(u^{S}, v^{S}\right)$ is the parameter coordinate corresponding to the integral point $k$ on the basic voxel, and $N^{S}$ is the basis function corresponding to the basic voxel. The difference between formulas (15) and (16) is in terms of form, $3 N_{\Gamma}$ becomes $3 N_{\Omega}$. For space problems, $N_{\Gamma}$ and $N_{\Omega}$, respectively, represent the number of basis functions in different parameter domains on the cutting surface of the transportation object in the supply chain. Finally, the solution of the force vector is converted from the original control points of the clipping plane of the supply chain transportation object to the control points formed by the model, and the equivalent loading of the force field is realized. The transformation of the three-dimensional model of the supply chain transportation object cutting can also be done in the same way. At this time, the parameter coordinates are transformed from $\left(u^{S}, v^{S}\right)$ to $\left(u^{V}, v^{V}, w^{V}\right)$.

In order to realize the program, we first find a series of corresponding integration points in the parameter space of the cutting boundary of the transportation object in the supply chain according to the Gauss quadrature rule. After that, we calculated the corresponding physical coordinates according to the cutting boundary spline formula of the objects transported in the supply chain, and finally the coordinates went through the process of parameter inversion to obtain the corresponding parameter coordinates. As 
shown in Figure 2, it provides a back-calculation process for the parameters of the cutting unit of transportation objects in the supply chain.

In order to verify the reliability of the proposed cutting algorithm for supply chain transportation objects, this example uses thicker supply chain transportation equipment (inner diameter $R_{i}=3 \mathrm{~mm}$, outer diameter $R_{0}=8 \mathrm{~mm}$ ), and supply chain transportation objects are cut into thinner cylinders. The parameters of the thick-walled barrel for supply chain transportation objects whose inner wall is subjected to uniform pressure are: inner diameter $R_{i}=4 \mathrm{~mm}$, outer diameter $R_{0}=7 \mathrm{~mm}$, axial length $h=10 \mathrm{~mm}$, internal pressure $=1 \mathrm{MPa}$, external pressure $P=0$, the modulus of elasticity $E=10^{5} \mathrm{MPa}$, and the Poisson's ratio $v=0.3$. The schematic diagram of the thick-walled cylinder calculation example is shown in Figure 3. Taking into account the symmetry of the problem, only the quarter circle is considered here, and the corresponding boundary conditions are given as shown in the figure.

Due to the analytical solution of this example, the theoretical calculation formula for the thick wall of the supply chain transportation equipment is as follows.

The displacement $u$ is

$$
u_{r}(r)=\frac{1}{E} \frac{P R_{i}^{2}}{R_{0}^{2}-R_{i}^{2}}\left((1-v) r+\frac{R_{0}^{2}(1+v)}{r}\right) .
$$

The radial virtual perception $\sigma_{r}$ is

$$
\sigma_{r}(r)=\frac{P R_{i}^{2}}{R_{0}^{2}-R_{i}^{2}}-\frac{P R_{i}^{2} R_{0}^{2}}{r^{2}\left(R_{0}^{2}-R_{i}^{2}\right)} .
$$

The circular virtual perception $\sigma_{\theta}$ is

$$
\sigma_{\theta}(r)=\frac{P R_{i}^{2}}{R_{0}^{2}-R_{i}^{2}}+\frac{P R_{i}^{2} R_{0}^{2}}{r^{2}\left(R_{0}^{2}-R_{i}^{2}\right)} .
$$

\section{Visual Simulation of Modern Supply Chain Based on MR Virtual Reality Technology}

The visual simulation of supply chain is realized by real-time dynamic display based on geometric model. The application of geometric model method in visual simulation is mainly to establish three-dimensional geometric model and kinematics model for independent entities (such as factory buildings, equipment, products, parts, etc.). Then, it uses these three-dimensional model components to replace various entities in the supply chain system, and then combines these independent entities to create a simulation model that simulates reality, and uses animation to display the simulation process and results during the simulation operation. The visual display system includes four parts: browsing of virtual objects, interactive establishment of visual simulation models, animation display of simulation running, and icon display of simulation statistical results. The visual simulation model and simulation animation depend on the virtual object model. The virtual object model is a prerequisite for the establishment of a visual simulation

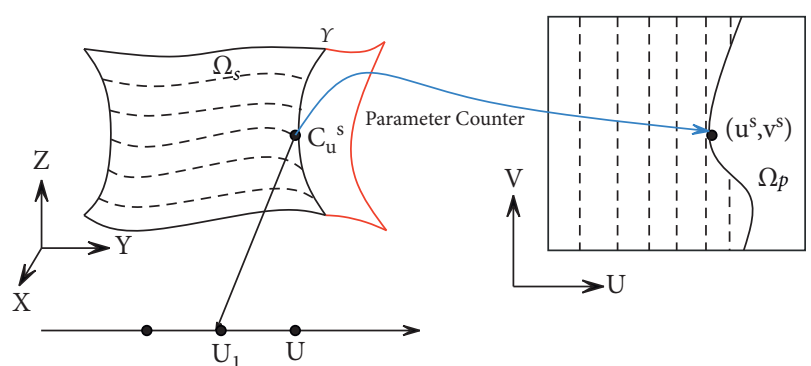

FIGURE 2: Back-calculation of cutting surface parameters of objects transported in the supply chain.

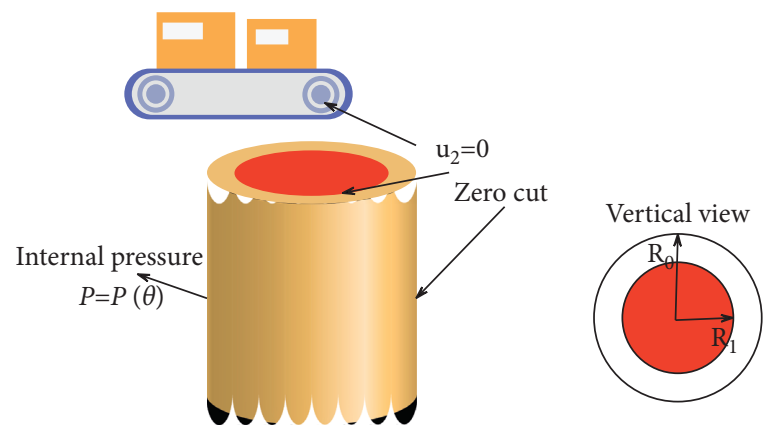

FIGURE 3: Internal pressurization of supply chain transportation equipment.

model of the supply chain and an important content of the visual display system. Virtual objects can be divided into three categories according to their operating conditions in the simulation experiment: movable objects, resource objects related to moving objects, and static environment objects, as shown in Figure 4.

The supply chain is a large complex system, and hierarchical display is the most natural design method for supply chain visualization. A typical supply chain consists of multiple interrelated production plants, each of which includes the input of raw materials, the output of finished products, and the processing process from raw materials to finished products. Moreover, each link of the product has to involve the process and equipment for customs clearance. According to the hierarchical structure of the supply chain, it can be realized from the macro to the specific, which can effectively express the various links of the supply chain and meet the different needs of users. Therefore, the hierarchical display is very conducive to effective supply chain visualization. The product-oriented supply chain is dominated by customer demand and centered on the production enterprise. Its visual display can be divided into three layers: the supply chain layer, the core enterprise layer, and the part manufacturing process layer. The information displayed at the supply chain layer includes the construction of the supply chain and the logistics operations among its members. The core enterprise layer expresses the production organization of the product, and the part manufacturing process layer shows the processing flow of the main parts. The display of the next layer is a refinement of a certain part of the previous layer. After integration, we can get a detailed display of the next layer by clicking the corresponding icon 


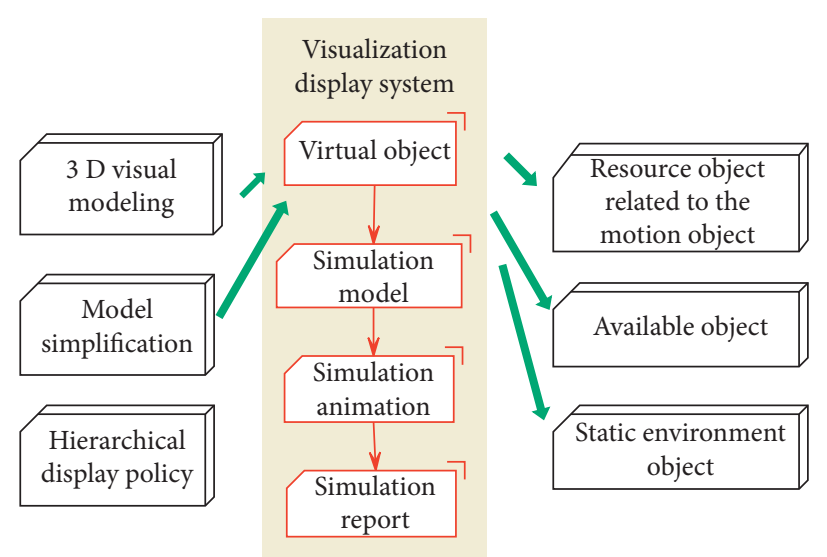

FIGURE 4: 3D visualization display and realization in simulation.

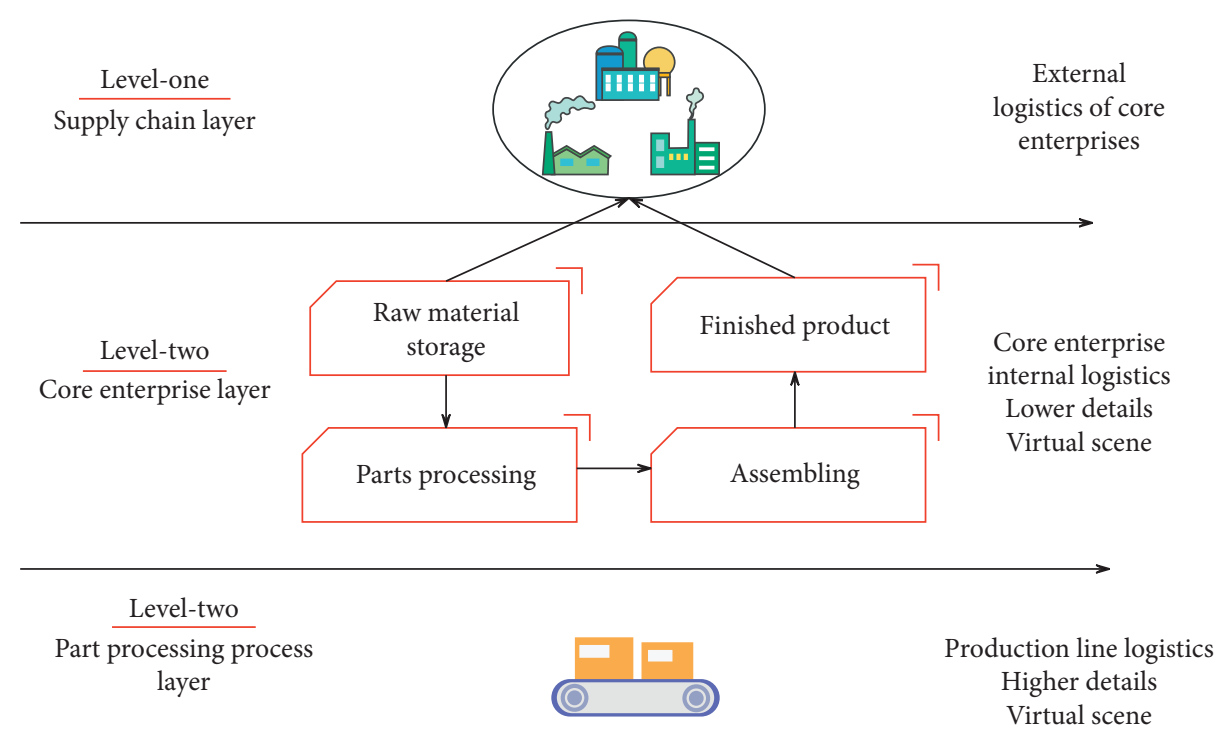

FIGURE 5: Visualization levels of the supply chain.

on the previous layer. The layer of visualization is shown in Figure 5.

The flow of the discrete event system simulation program is shown in Figure 6. The key is to determine the sequence of all events according to the time sequence in which they occurred. Except for the initial events, the events in the event sequence cannot be determined in advance before the simulation, but are generated during the simulation. In discrete event simulation, it is generally used to plan the event in advance, that is, the event must have been planned before the simulation system processes any event. The main work of planning is to determine the type and time of the event. The planned event is stored in the event table. When the simulation clock reaches the time when the event is generated, the simulation system will process the event.

The supply chain visual simulation system is implemented based on the QUEST simulation tool, and the system structure is shown in Figure 7.

When constructing the modeling object of the supply chain system, the intelligent perception algorithm used in the third part needs to be used. It predefines the geometric characteristics and kinematics characteristics of the modeling object, then encapsulates these characteristics into the object class, defines the behavior characteristics, and builds the object class library. Users can also encapsulate self-edited programs into object class attributes for logical control, and then establish logical connections between objects to control logistics operations, thereby establishing a system simulation model. The establishment process of the visual simulation model of the supply chain is shown in Figure 8.

The server architecture of the system is a traditional layered architecture, which includes Gateway, Controller, Service, Repository, Advise, etc., as shown in Figure 9.

After constructing a modern supply chain visual simulation system based on MR virtual reality technology, the system in this paper is verified. According to the actual needs of supply chain visual simulation, this paper constructs a supply chain system through a simulation platform, and verifies the effects of transportation object simulation, logistics route simulation, and information transfer simulation of the supply chain system in this paper, and calculates the test results. The results are shown in Figures 10-12. 


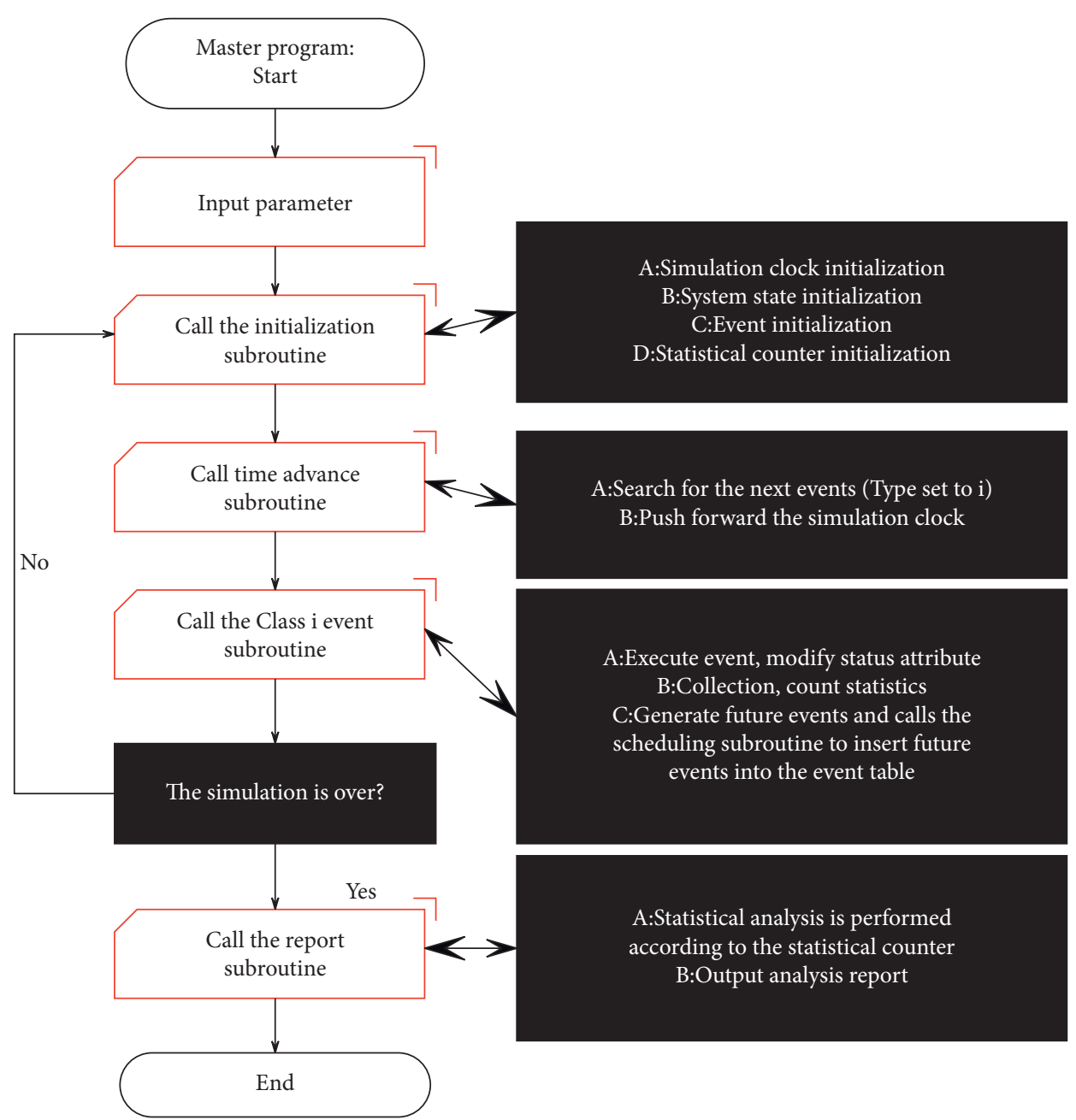

Figure 6: The structure diagram of the flow of the discrete event system simulation program.
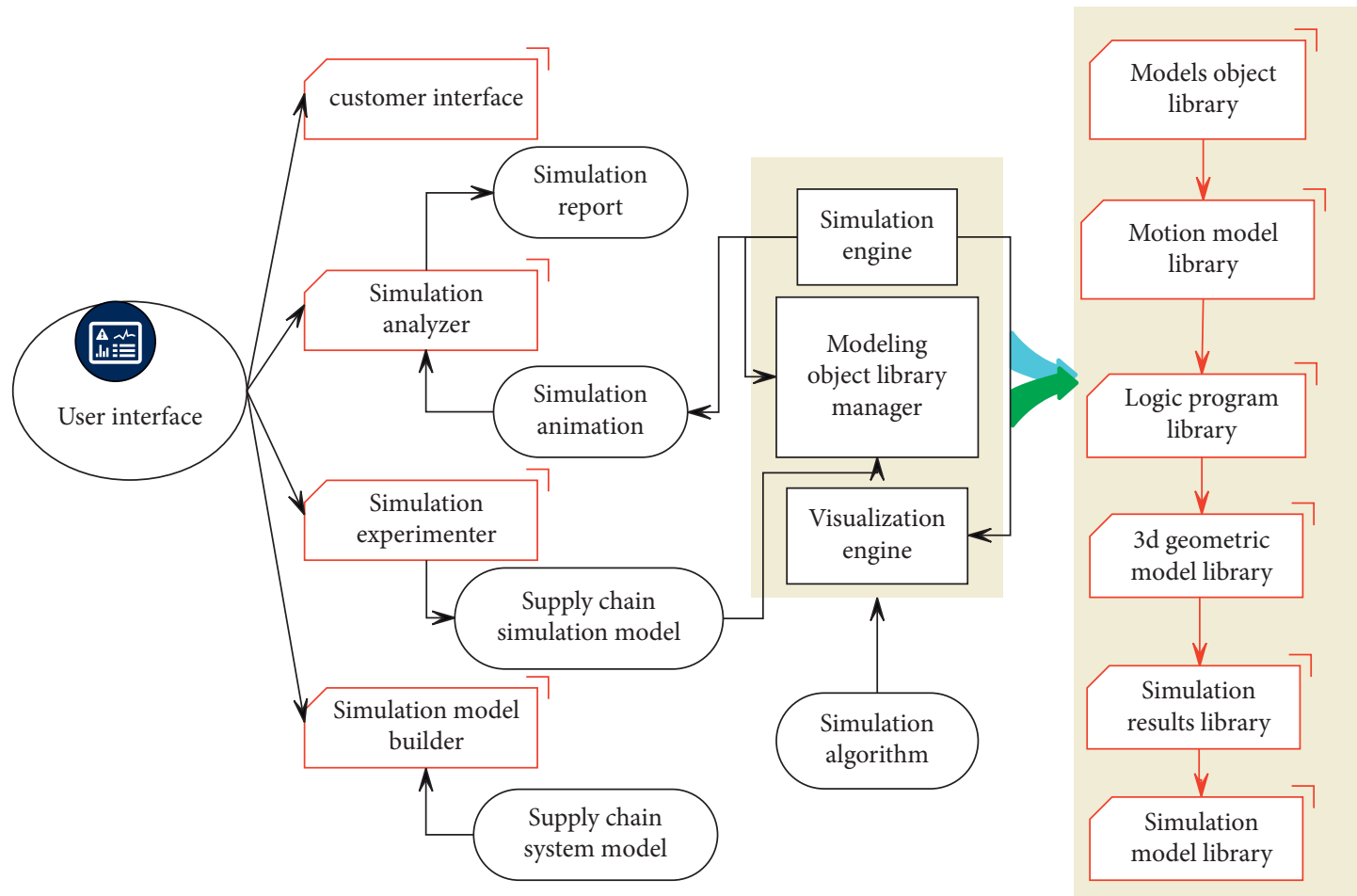

FIGURE 7: The architecture of the supply chain visual simulation system. 


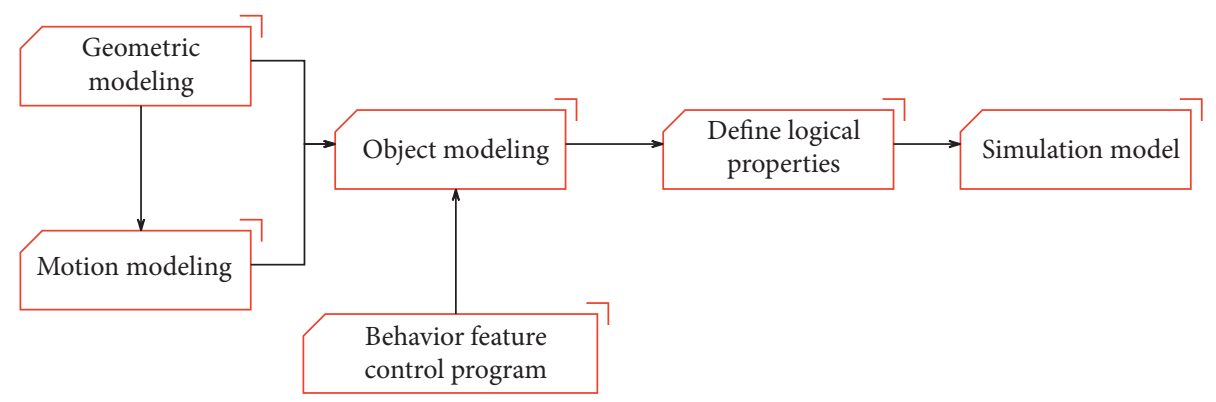

FIGURE 8: The process of establishing a simulation model.

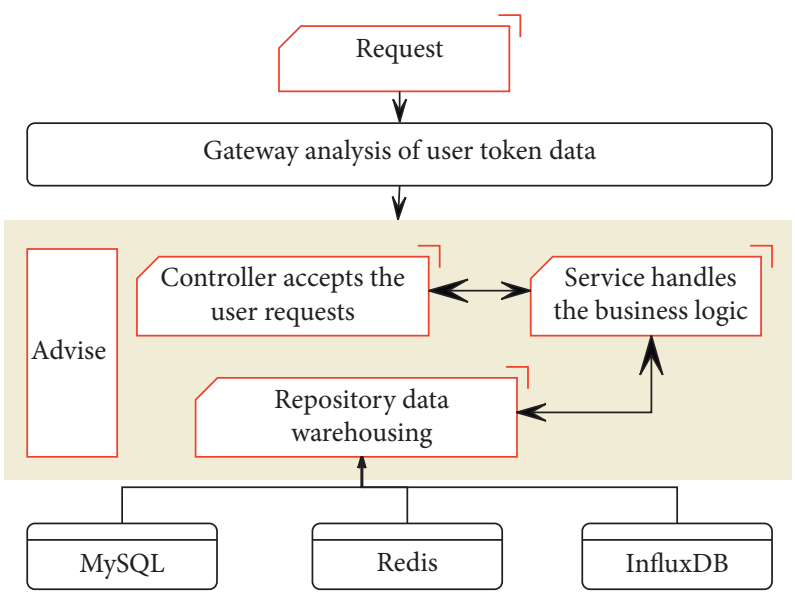

Figure 9: Server-side architecture.

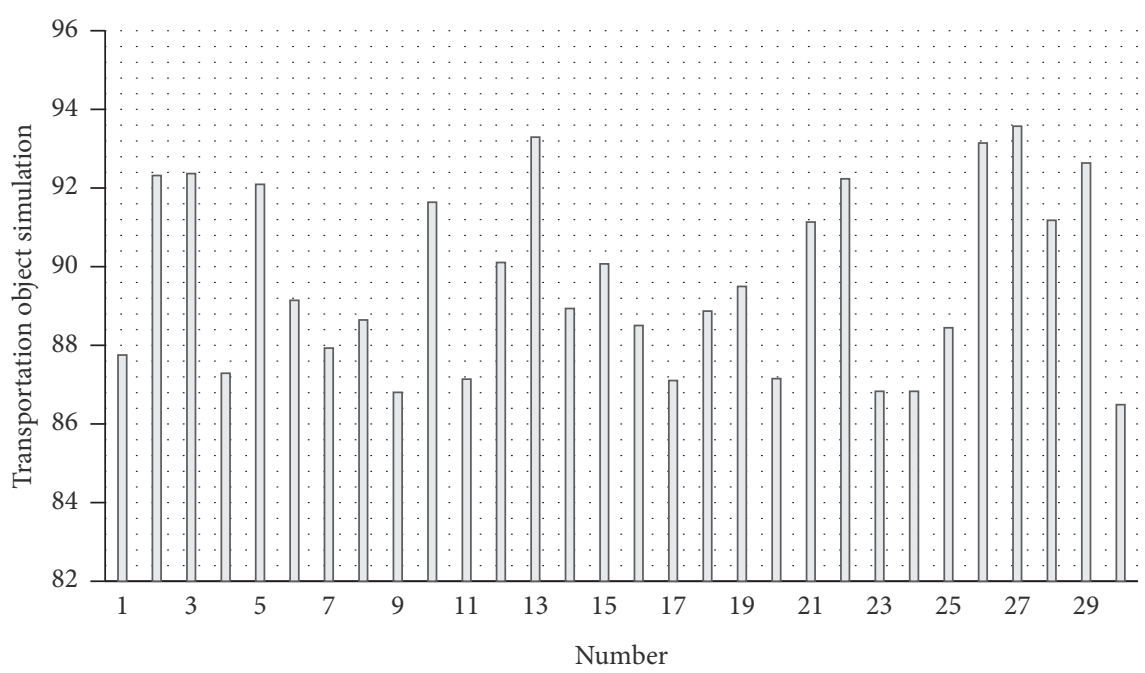

Figure 10: Transportation objects simulation. 


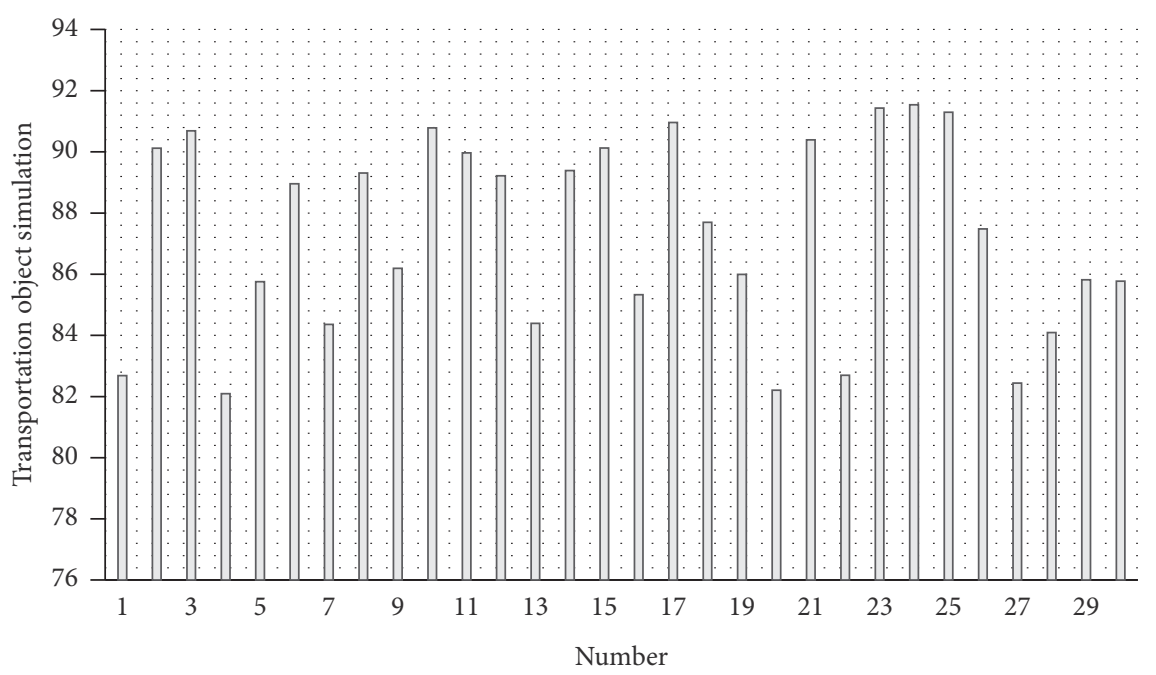

Figure 11: Logistics route simulation.

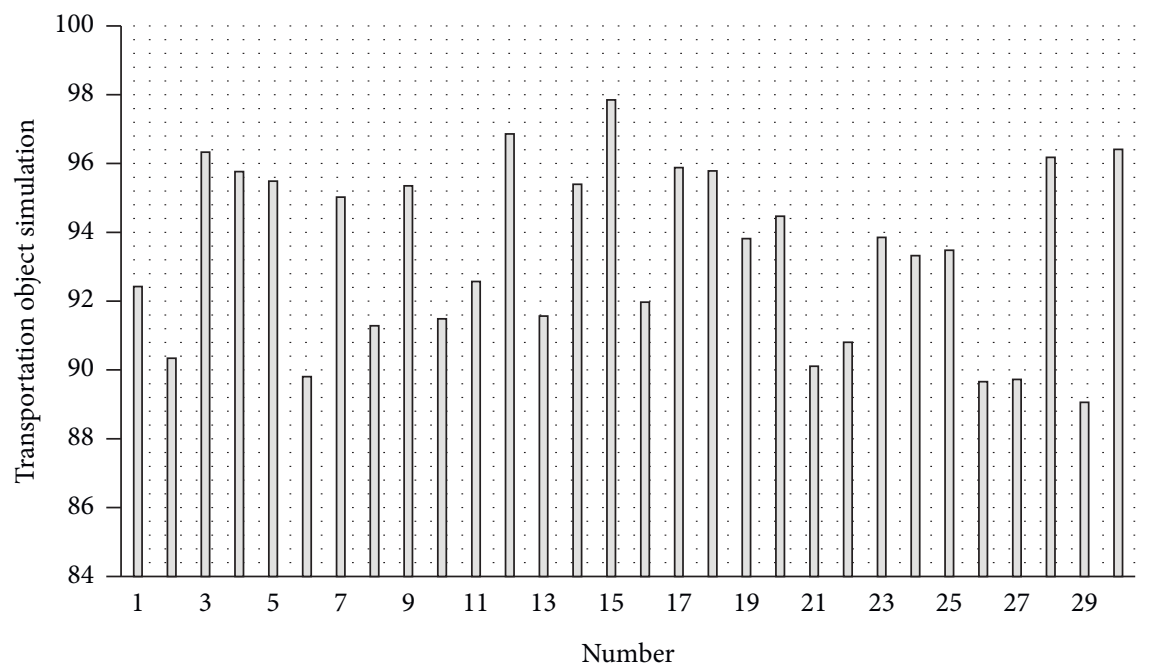

FIGURE 12: Information transfer simulation.

Through the above experimental research, this paper verifies that the modern supply chain visual simulation technology based on MR virtual reality technology has a good simulation effect in the supply chain system.

\section{Conclusion}

There are many kinds of information in the supply chain, and the value of information flow is of great significance to the development and management of enterprises. The biggest problem facing the supply chain today is how to manage the supply chain member companies so that they can obtain consistent information and take consistent actions to achieve coordination within the entire enterprise group. In the process of decentralized control, supply chain member companies always make their own decisions optimal without considering the impact of their decisions on other companies and the efficiency of the entire supply chain. This paper combines the MR virtual reality technology to carry out the visual simulation of the modern supply chain, improve the management and operation efficiency of the modern supply chain, and provide a theoretical reference for the subsequent research on the visual management of the supply chain. The experimental research verifies that the modern supply chain visual simulation technology based on MR virtual reality technology has a good simulation effect in the supply chain system.

\section{Data Availability}

The labeled dataset used to support the findings of this study is available from the corresponding author upon request.

\section{Conflicts of Interest}

The author declares no conflicts of interest. 


\section{Acknowledgments}

This study was sponsored by Research on the Mixed Reality (MR) Holographic Teaching Application of Virtual Reloading Technology in Modern Logistics Node.

\section{References}

[1] A. Pal and K. Kant, "Using blockchain for provenance and traceability in internet of things-integrated food logistics," Computer, vol. 52, no. 12, pp. 94-98, 2019.

[2] T. Qu, M. Thürer, J. Wang et al., "System dynamics analysis for an Internet-of-Things-enabled production logistics system," International Journal of Production Research, vol. 55, no. 9, pp. 2622-2649, 2017.

[3] Y. Ding, M. Jin, S. Li, and D. Feng, "Smart logistics based on the internet of things technology: an overview," International Journal of Logistics Research and Applications, vol. 24, no. 4, pp. 323-345, 2021.

[4] Y. Gu and Q. Liu, "Research on the application of the internet of things in reverse logistics information management," Journal of Industrial Engineering and Management, vol. 6, no. 4, pp. 963-973, 2013.

[5] J. Chen and W. Zhao, "Logistics automation management based on the Internet of things," Cluster Computing, vol. 22, no. 6, pp. 13627-13634, 2019.

[6] S. Huang, Y. Guo, S. Zha, and Y. Wang, "An internet-ofthings-based production logistics optimisation method for discrete manufacturing," International Journal of Computer Integrated Manufacturing, vol. 32, no. 1, pp. 13-26, 2019.

[7] S. Yadav, D. Garg, and S. Luthra, "Selection of third-party logistics services for internet of things-based agriculture supply chain management," International Journal of Logistics Systems and Management, vol. 35, no. 2, pp. 204-230, 2020.

[8] Y. P. Tsang, C. H. Wu, H. Y. Lam, K. L. Choy, and G. T. S. Ho, "Integrating Internet of Things and multi-temperature delivery planning for perishable food E-commerce logistics: a model and application," International Journal of Production Research, vol. 59, no. 5, pp. 1534-1556, 2021.

[9] S. Cho and J. Kim, "Smart logistics model on internet of things environment," Advanced Science Letters, vol. 23, no. 3, pp. 1599-1602, 2017.

[10] V. Kupriyanovsky, V. Alenkov, A. Stepanenko et al., "On development of transport and logistics industries in the European Union: open BIM, Internet of Things and cyberphysical systems," International Journal of Open Information Technologies, vol. 6, no. 2, pp. 54-100, 2018.

[11] M. Thürer, Y. H. Pan, T. Qu, H. Luo, C. D. Li, and G. Q. Huang, "Internet of Things (IoT) driven kanban system for reverse logistics: solid waste collection," Journal of Intelligent Manufacturing, vol. 30, no. 7, pp. 2621-2630, 2019.

[12] Y. Sun, H. Yan, C. Lu, R. Bie, and P. Thomas, "A holistic approach to visualizing business models for the internet of things," Communications in Mobile Computing, vol. 1, no. 1, pp. 1-7, 2012.

[13] R. Davis, M. Vochozka, J. Vrbka, and O. Neguriţă, "Industrial artificial intelligence, smart connected sensors, and big datadriven decision-making processes in Internet of Things-based real-time production logistics," Economics, Management, and Financial Markets, vol. 15, no. 3, pp. 9-15, 2020.

[14] C. Yang, W. Shen, and X. Wang, "The internet of things in manufacturing: key issues and potential applications," IEEE Systems, Man, and Cybernetics Magazine, vol. 4, no. 1, pp. 6-15, 2018.
[15] K. Coatney and M. Poliak, "Cognitive decision-making algorithms, internet of things smart devices, and sustainable organizational performance in Industry 4.0-based manufacturing systems," Journal of Self-Governance and Management Economics, vol. 8, no. 4, pp. 9-18, 2020.

[16] M. Hawkins, "Cyber-physical production networks, internet of things-enabled sustainability, and smart factory performance in industry 4.0-based manufacturing systems," Economics, Management, and Financial Markets, vol. 16, no. 2, pp. 73-83, 2021.

[17] C. Kalaivani and G. Indhumathi, "Application OF internet OF things (iot) IN logistics industry," IJRAR-International Journal of Research and Analytical Reviews (IJRAR), vol. 5, no. 3, pp. 114-118, 2018.

[18] J. A. J. Alsayaydeh, "Stratified model of the internet of things infrastructure* jamil abedalrahim jamil alsayaydeh, "vadym shkarupylo," mohd saad bin Hamid,"Stepan skrupsky and" andrii oliinyk" fakulti teknologi Kejuruteraan, "Center for advanced computing technology, universiti teknikal Malaysia melaka," Journal of Engineering and Applied Sciences, vol. 13, no. 20, pp. 8634-8638, 2018.

[19] H. Yang, S. Kumara, S. T. S. Bukkapatnam, and F. Tsung, "The internet of things for smart manufacturing: a review," IISE Transactions, vol. 51, no. 11, pp. 1190-1216, 2019.

[20] V. Suma, "Towards sustainable industrialization using big data and internet of things," Journal of ISMAC, vol. 1, no. 1, pp. 24-37, 2019.

[21] G. Knieps, "Internet of things, future networks, and the economics of virtual networks," Competition and Regulation in Network Industries, vol. 18, no. 3-4, pp. 240-255, 2017. 